\title{
Article \\ Clinicopathological and Molecular Features of Patients with Early and Late Recurrence after Curative Surgery for Colorectal Cancer
}

\author{
Yuan-Tzu Lan ${ }^{1,2, *}$, Shih-Ching Chang ${ }^{1,2} \mathbb{D}$, Pei-Ching Lin ${ }^{3,4}$, Chun-Chi Lin ${ }^{1,2} \mathbb{D}$, Hung-Hsin Lin ${ }^{1,2} \mathbb{D}$, \\ Sheng-Chieh Huang ${ }^{1,2}$, Chien-Hsing Lin ${ }^{5}$, Wen-Yi Liang ${ }^{6}$, Wei-Shone Chen ${ }^{1,2}$, Jeng-Kai Jiang ${ }^{1,2}$, \\ Shung-Haur Yang ${ }^{1,2,7}$ and Jen-Kou Lin ${ }^{1,2}$
}

1 Division of Colon \& Rectal Surgery, Department of Surgery, Taipei Veterans General Hospital, Taipei 11217, Taiwan; changsc@vghtpe.gov.tw (S.-C.C.); cclin15@vghtpe.gov.tw (C.-C.L.); hhlin7@vghtpe.gov.tw (H.-H.L.); schuang5@vghtpe.gov.tw (S.-C.H.); wschen@vghtpe.gov.tw (W.-S.C.); jkjiang@vghtpe.gov.tw (J.-K.J.); yangsh@vghtpe.gov.tw (S.-H.Y.); jklin@vghtpe.gov.tw (J.-K.L.)

2 Department of Surgery, Faculty of Medicine, School of Medicine, National Yang Ming Chiao Tung University, Taipei 112, Taiwan

3 Department of Clinical Pathology, Yang-Ming Branch, Taipei City Hospital, Taipei 11146, Taiwan; b7901127@tmu.edu.tw

4 Department of Health and Welfare, University of Taipei, Taipei 11153, Taiwan

5 Division of Genomic Medicine, National Health Research Institutes, Zhunan 35053, Taiwan; jameslin@fcbiotech.com.tw

check for updates

Citation: Lan, Y.-T.; Chang, S.-C.; Lin, P.-C.; Lin, C.-C.; Lin, H.-H.; Huang, S.-C.; Lin, C.-H.; Liang, W.-Y.; Chen, W.-S.; Jiang, J.-K.; et al. Clinicopathological and Molecular Features of Patients with Early and Late Recurrence after Curative Surgery for Colorectal Cancer. Cancers 2021, 13, 1883. https:// doi.org/10.3390/cancers13081883

Academic Editor: Matteo Donadon

Received: 5 March 2021

Accepted: 12 April 2021

Published: 14 April 2021

Publisher's Note: MDPI stays neutral with regard to jurisdictional claims in published maps and institutional affiliations.

Copyright: () 2021 by the authors. Licensee MDPI, Basel, Switzerland. This article is an open access article distributed under the terms and conditions of the Creative Commons Attribution (CC BY) license (https:// creativecommons.org/licenses/by/ $4.0 /)$.
6 Department of Pathology, Taipei Veterans General Hospital, Taipei 11217, Taiwan; wyliang@vghtpe.gov.tw

7 Department of Surgery, National Yang Ming Chiao Tung University Hospital, Yilan 26058, Taiwan

* Correspondence: ytlan@vghtpe.gov.tw; Tel.: +886-2-2875-7544-110; Fax: +886-2-2875-7639

Simple Summary: Colorectal cancer patients with early recurrence had advanced pathological node categories, pathological tumor, node, metastasis stages, adjuvant chemotherapy treatment, a worse overall survival rate, more liver metastases and more APC mutations than those with late recurrence. Patients with right-sided colon cancer tended to have early recurrence than those with left-sided colon cancer or rectal cancer. The postrecurrence survival rates were not significantly different between patients with early and late recurrence, which was observed in right-sided colon, left-sided colon and rectum. Multivariate analysis showed that old age, early recurrence, multiple-site recurrence and $B R A F$ and NRAS mutations were independent prognostic factors.

Abstract: Background: Few reports have investigated genetic alterations between patients with early and late recurrence following curative surgery for colorectal cancer (CRC). Methods: A total of 1227 stage I-III CRC patients who underwent curative resection were included retrospectively. Among them, 236 patients had tumor recurrence: 139 had early ( $<2$ years after surgery) and 97 had late ( $\geq 2$ years after surgery) recurrence. Clinicopathological features and genetic alterations were compared between the two groups. Results: Compared to those with late recurrence, patients with early recurrence were more likely to have advanced pathological node $(\mathrm{N})$ categories; tumor, node, metastasis (TNM) stages; adjuvant chemotherapy treatment; liver metastases; APC mutations; and worse five-year overall survival rates. Patients with right-sided colon cancer were more likely to develop early recurrence than were those with left-sided colon cancer or rectal cancer. Regarding rectal cancer, patients with early recurrence were more likely to be at advanced pathological $\mathrm{N}$ categories and TNM stages than those with late recurrence. Multivariate analysis revealed old age, early recurrence, multiple-site recurrence, and $B R A F$ and NRAS mutations to be independent prognostic factors. Conclusion: CRC patients with early recurrence have a worse OS rate and more $A P C$ mutations than those with late recurrence.

Keywords: colorectal cancer; early recurrence; late recurrence; prognostic factor; genetic alteration 


\section{Introduction}

Approximately 1.8 million new cases of colorectal cancer (CRC) are diagnosed annually, making it the third most commonly diagnosed malignancy and the second-leading cause of cancer deaths worldwide [1]. In Taiwan, colorectal cancer (CRC) is the most common cancer type and the third leading cause of cancer-related death [2]. As CRC accounts for approximately 15,000 new cancer diagnoses and 5700 cancer-related deaths every year, it is a major public health problem. It has been reported that the recurrence rate following curative surgery for CRC is $26-31 \%$, with recurrence within five years after surgery in most cases $[3,4]$.

The definition of the time period for early recurrence varies among studies, ranging from one to three years after surgery [5-8]. Although it has been reported that CRC patients with early recurrence have worse survival than those with late recurrence, no significant difference in the clinicopathological features was observed between those with early and late recurrence [6]. Nonetheless, KRAS mutation is more common in CRC patients with early tumor recurrence [6]. In our previous study [7], patients with multiple organ recurrence tended to die within two years after recurrence. However, differences in genetic alterations between CRC patients with single-site recurrence and those with multiple-site recurrence remain unclear.

To date, there have been few reports regarding the correlation between mutational profiles, recurrence pattern, and prognosis of CRC patients with early and late recurrence. Hence, we sought to determine whether the molecular profiles of patients with early and late recurrence differ, which might explain the difference in prognosis between these two groups. The aim of the present study was to compare differences in clinicopathological and mutational profiles between CRC patients with early and late recurrence.

\section{Materials and Methods}

\subsection{Patient Enrollment}

Between 2000 and 2010, a total of 1227 stage I-III CRC patients who underwent curative resection and for whom tumor samples stored in the biobank of Taipei Veterans General Hospital were available were included in this retrospective study. Among them, 236 patients (19.5\%) had tumor recurrence following surgery: Of these, 139 had early recurrence and 97 had late recurrence. Early recurrence was defined as tumor recurrence diagnosed within two years after surgery; late recurrence was defined as tumor recurrence diagnosed two years or more after surgery. Clinicopathological features and genetic alterations were compared between the two groups. Written informed consent for sample collection and storage in the biobank was provided by all enrolled patients. The present study was approved by the Institutional Review Board of Taipei Veterans General Hospital.

Patients who received preoperative chemoradiotherapy or palliative surgery, who did not receive surgical treatment for primary CRC, who did not have available tumor tissue in the biobank, who underwent emergent operations, or who died within 30 days after surgery were excluded. Right-sided colon cancer was defined as a tumor located from the cecum to the transverse colon; left-sided colon cancer was defined as a tumor extending from the splenic flexure to the sigmoid colon.

After surgery, patients were followed up every three months for the first two years and every six months thereafter. In addition, carcinoembryonic antigen analysis, abdominal sonogram, chest radiography, and abdominal computerized tomography were arranged if needed. Proton emission tomography or magnetic resonance imaging was performed when the carcinoembryonic antigen level was elevated without confirmation of the site of tumor recurrence. Tumor recurrence was evaluated according to the following variables: timing (early/ $<2$ years and late/ $>2$ years), site (local, liver, lung, peritoneum, bone, and other sites) and number of sites (single site or multiple sites). Patients with resectable synchronous or metachronous metastasis were treated with surgery and adjuvant chemotherapy based on folinic acid, fluorouracil, and oxaliplatin (FOLFOX). Targeted therapies such as be- 
vacizumab, cetuximab, and panitumumab were not reimbursed by the Taiwan National Health Insurance Administration before 2010.

\subsection{DNA Extraction and Mutational Analysis of a 12-Gene Panel}

DNA extraction was performed using a QIAamp DNA Tissue Kit (Qiagen, Valencia, CA, USA). A 12-gene panel for the identification of 139 mutations selected from among hotspots was assessed according to the Catalog Of Somatic Mutations In Cancer (COSMIC) database and previous studies $[9,10]$. As described in a previous report [11], the MassArray method was used to detect 139 hotspot mutations in 12 genes.

\subsection{Microsatellite Instability (MSI) Analysis}

Five microsatellite markers; namely, D5S345, D2S123, BAT25, BAT26, and D17S250, were used to define the MSI phenotype according to international criteria [12]. MSI-high tumors were defined as those with two or more positive MSI markers; microsatellite stable (MSS) tumors were defined as those with zero or one positive MSI marker.

Mutational analysis of a 12-gene panel and MSI analysis were performed on tissue specimens of primary CRC stored in the biobank of Taipei Veterans General Hospital. Biopsy tissues of recurrent tumors were not analyzed for mutational and MSI profiles. The investigators were unaware of the clinical outcome of the patients when analyzing the genetic mutations and MSI phenotype.

\subsection{Statistical Analysis}

IBM SPSS Statistics 25.0 (IBM Corp., Armonk, NY, USA) was used to perform the statistical analyses. Clinicopathological features were compared using chi-squared and twotailed Fisher's exact tests. Numerical values were compared using Student's $t$-test. Overall survival (OS) was considered as the time from the date of diagnosis to the date of death. Kaplan-Meier survival curves were compared using the log-rank test. Univariate and multivariate Cox regression analyses were employed to assess the impact of molecular and clinicopathological features on OS. Variables differing significantly in univariate analysis were included in multivariate analysis based on logistic regression. Tumor recurrence was the primary endpoint, and OS was the secondary endpoint. A $p$ value less than 0.05 was defined as statistically significant.

\section{Results}

\subsection{Clinicopathological Features and Genetic Alterations}

Among the 236 CRC patients who experienced tumor recurrence after curative surgery, $139(58.9 \%)$ with recurrence within two years were considered the early recurrence group, whereas 97 patients with recurrence $\geq 2$ years following surgery were considered the laterecurrence group. The follow-up time was $48.4 \pm 46.0$ months and $80.0 \pm 45.5$ months in the early- and late-recurrence groups, respectively. In the early-recurrence group, the median recurrence time was 12.5 months and the median overall survival time was 32.1 months. In the late-recurrence group, the median recurrence time was 40.4 months and the median overall survival time was 65.7 months. All variables reported in the first column of Tables 1 and 2 refer to primary colorectal cancer. As shown in Table 1, compared to patients with late recurrence, those with early recurrence were more likely to be at advanced pathological $\mathrm{N}$ categories and TNM stages, to have undergone adjuvant chemotherapy, and to carry APC mutations. Additionally, patients with right-sided colon cancer were more likely to develop early recurrence than those with left-sided colon cancer or rectal cancer. Because the high variance inflation factor was more than 1.5 , we excluded the pathologic $\mathrm{N}$ category in multiple testing correction logistic regression analysis. Ultimately, four covariates (tumor location, adjuvant chemotherapy, pathological TNM stage, and APC mutations) were included in this analysis, which demonstrated that patients with early recurrence were more likely to have right-sided colon cancer, be at advanced pathological TNM stages, and harbor $A P C$ mutations than patients with late recurrence. The Cramar value is provided in 
Table 1. The variance inflation factor was 1.02 and the Bayesian information criterion was -968.836 .

Table 1. Clinicopathological features and mutation spectrum between early and late recurrence after curative surgery for colorectal cancer.

\begin{tabular}{|c|c|c|c|c|c|c|c|}
\hline \multirow[b]{2}{*}{ Variables } & \multicolumn{4}{|c|}{ Univariate Analysis } & \multicolumn{3}{|c|}{$\begin{array}{c}\text { Multiple Testing Correction Logistic } \\
\text { Regression }\end{array}$} \\
\hline & $\begin{array}{c}\text { Early } \\
\text { Recurrence } \\
n=139 \\
n(\%)\end{array}$ & $\begin{array}{c}\text { Late } \\
\text { Recurrence } \\
n=97 \\
n(\%)\end{array}$ & $\begin{array}{l}\text { Cramer } \\
\text { Value }\end{array}$ & $p$ Value & $\begin{array}{l}\text { Odds } \\
\text { Ratio }\end{array}$ & $\begin{array}{l}\text { Confidence } \\
\text { Interval }\end{array}$ & $p$ Value \\
\hline Age (years) & & & 0.043 & 0.508 & & & \\
\hline$<70$ & $72(51.8)$ & $46(47.4)$ & & & & & \\
\hline$\geq 70$ & $67(48.2)$ & $51(52.6)$ & & & & & \\
\hline$\overline{S e x}$ & & & 0.045 & 0.489 & & & \\
\hline Male & $90(64.7)$ & $67(69.1)$ & & & & & \\
\hline Female & $49(35.3)$ & $30(30.9)$ & & & & & \\
\hline Tumor location & & & 0.199 & 0.009 & & & 0.015 \\
\hline Right-sided colon & $40(28.8)$ & $13(13.4)$ & & & 1.000 & & \\
\hline Left-sided colon & $37(26.6)$ & $39(40.2)$ & & & 3.225 & $1.451-7.168$ & \\
\hline Rectum & $62(44.6)$ & $45(46.4)$ & & & 2.380 & $1.105-5.124$ & \\
\hline Tumor differentiation & & & 0.088 & 0.174 & & & \\
\hline Well to moderate & $129(92.8)$ & $94(96.9)$ & & & & & \\
\hline Poor & $10(7.2)$ & $3(3.1)$ & & & & & \\
\hline Lymphovascular invasion & $37(26.6)$ & $19(19.6)$ & 0.081 & 0.212 & & & \\
\hline Adjuvant chemotherapy & $94(67.6)$ & $48(49.5)$ & 0.182 & 0.005 & & & \\
\hline Pathological T category & & & 0.088 & 0.605 & & & \\
\hline $\mathrm{T} 1$ & $1(0.7)$ & $1(1.0)$ & & & & & \\
\hline $\mathrm{T} 2$ & $11(7.9)$ & $9(9.3)$ & & & & & \\
\hline T3 & $106(76.3)$ & $78(80.4)$ & & & & & \\
\hline $\mathrm{T} 4$ & $21(15.1)$ & $9(9.3)$ & & & & & \\
\hline Pathological N category & & & 0.223 & 0.003 & & & 0.005 \\
\hline N0 & $40(28.8)$ & $47(48.5)$ & & & 1.000 & & \\
\hline N1 & $42(30.2)$ & $28(28.9)$ & & & 0.539 & $0.278-1.045$ & \\
\hline N2 & $57(41.0)$ & $22(22.7)$ & & & 0.336 & $0.172-0.656$ & \\
\hline Pathological TNM stage & & & 0.201 & 0.008 & & & \\
\hline I & $6(4.3)$ & $8(8.2)$ & & & & & \\
\hline II & $34(24.5)$ & $39(40.2)$ & & & & & \\
\hline III & $99(71.2)$ & $50(51.5)$ & & & & & \\
\hline MSI status & & & 0.055 & 0.398 & & & \\
\hline MSS & $129(92.8)$ & 87 (89.7) & & & & & \\
\hline MSI-high & $10(7.2)$ & $10(10.3)$ & & & & & \\
\hline \multicolumn{8}{|l|}{ Genetic mutations } \\
\hline TP53 & $49(35.3)$ & $31(32.0)$ & 0.034 & 0.599 & & & \\
\hline$A P C$ & $42(30.2)$ & $18(18.6)$ & 0.132 & 0.043 & 0.462 & $0.237-0.898$ & 0.023 \\
\hline PIK3CA & $24(17.3)$ & $11(11.3)$ & 0.082 & 0.208 & & & \\
\hline$B R A F$ & $7(5.0)$ & $3(3.1)$ & 0.047 & 0.466 & & & \\
\hline$K R A S$ & $51(36.7)$ & $39(40.2)$ & 0.036 & 0.584 & & & \\
\hline NRAS & $13(9.4)$ & $5(5.2)$ & 0.078 & 0.232 & & & \\
\hline HRAS & $4(2.9)$ & $3(3.1)$ & 0.006 & 0.924 & & & \\
\hline FBXW7 & $8(5.8)$ & $4(4.1)$ & 0.037 & 0.575 & & & \\
\hline PTEN & $1(0.7)$ & 0 & 0.054 & 0.403 & & & \\
\hline SMAD4 & $5(3.6)$ & $1(1.0)$ & 0.218 & 0.218 & & & \\
\hline TGF $\beta$ & $3(2.2)$ & $1(1.0)$ & 0.043 & 0.509 & & & \\
\hline AKT1 & $1(0.7)$ & $1(1.0)$ & 0.017 & 0.797 & & & \\
\hline
\end{tabular}

MSI: microsatellite instability; MSS: microsatellite stable; TNM: tumor, node, metastasis; bold: statistically significant. 
Table 2. Clinicopathological features between early and late recurrence of colorectal cancer according to tumor location.

\begin{tabular}{|c|c|c|c|c|c|c|c|c|c|}
\hline \multirow[b]{2}{*}{ Variables } & \multicolumn{3}{|c|}{ Right-Sided Colon Cancer } & \multicolumn{3}{|c|}{ Left-Sided Colon Cancer } & \multicolumn{3}{|c|}{ Rectal Cancer } \\
\hline & $\begin{array}{c}\text { Early } \\
\text { Recurrence } \\
n=40 \\
n(\%)\end{array}$ & $\begin{array}{c}\text { Late } \\
\text { Recurrence } \\
n=13 \\
n(\%)\end{array}$ & $p$ Value & $\begin{array}{c}\text { Early } \\
\text { Recurrence } \\
n=37 \\
n(\%)\end{array}$ & $\begin{array}{c}\text { Late } \\
\text { Recurrence } \\
n=39 \\
n(\%)\end{array}$ & $p$ Value & $\begin{array}{c}\text { Early } \\
\text { Recurrence } \\
n=62 \\
n(\%)\end{array}$ & $\begin{array}{c}\text { Late } \\
\text { Recurrence } \\
n=45 \\
n(\%)\end{array}$ & $p$ Value \\
\hline Age (years) & & & 0.679 & & & 0.985 & & & 0.288 \\
\hline$<70$ & $18(45.0)$ & $5(38.5)$ & & $20(54.1)$ & $21(53.8)$ & & $34(54.8)$ & $20(44.4)$ & \\
\hline$\geq 70$ & $22(55.0)$ & $8(61.5)$ & & $17(45.9)$ & $18(46.2)$ & & $28(45.2)$ & $25(55.6)$ & \\
\hline$\overline{\text { Sex }}$ & & & 0.942 & & & 0.354 & & & 0.722 \\
\hline Male & $22(55.0)$ & $7(53.8)$ & & $26(70.3)$ & $31(79.5)$ & & $42(67.7)$ & $29(64.4)$ & \\
\hline Female & $18(45.0)$ & $6(46.2)$ & & $11(29.7)$ & $8(20.5)$ & & $20(32.3)$ & $16(35.6)$ & \\
\hline Tumor differentiation & & & 0.391 & & & 0.600 & & & - \\
\hline Well to moderate & $33(82.5)$ & $12(92.3)$ & & $34(91.9)$ & $37(94.9)$ & & $62(100)$ & $45(100)$ & \\
\hline Poor & $7(17.5)$ & $1(7.7)$ & & $3(8.1)$ & $2(5.1)$ & & 0 & 0 & \\
\hline Lymphovascular invasion & & & 0.860 & & & 0.120 & & & 0.288 \\
\hline Absent & $33(82.5)$ & $11(84.6)$ & & $25(67.6)$ & $31(79.5)$ & & $44(71.0)$ & $36(80.0)$ & \\
\hline Present & 7 (17.5) & $2(15.4)$ & & $12(32.4)$ & $8(20.5)$ & & $18(29.0)$ & $9(20.0)$ & \\
\hline Adjuvant chemotherapy & & & 0.349 & & & 0.246 & & & 0.050 \\
\hline No & $10(25.0)$ & $5(38.5)$ & & $15(40.5)$ & $21(53.8)$ & & $20(32.3)$ & $28(51.1)$ & \\
\hline Yes & $30(75.0)$ & $8(61.5)$ & & $22(59.5)$ & $18(46.2)$ & & $42(67.7)$ & $22(48.9)$ & \\
\hline Pathological T category & & & 0.747 & & & 0.452 & & & 0.678 \\
\hline $\mathrm{T} 1$ & 0 & 0 & & 0 & $1(2.6)$ & & $1(1.6)$ & 0 & \\
\hline $\mathrm{T} 2$ & $2(5.0)$ & $1(7.7)$ & & $1(2.7)$ & $3(7.7)$ & & $8(12.9)$ & $5(11.1)$ & \\
\hline T3 & $31(77.5)$ & $10(76.9)$ & & $32(86.5)$ & $33(84.6)$ & & $43(69.4)$ & $35(77.8)$ & \\
\hline $\mathrm{T} 4$ & $7(17.5)$ & $2(15.4)$ & & $4(10.8)$ & $2(5.1)$ & & $10(16.1)$ & $5(11.1)$ & \\
\hline Pathological N category & & & 0.083 & & & 0.592 & & & 0.020 \\
\hline No & $10(25.0)$ & $5(38.4)$ & & $12(32.4)$ & $17(43.6)$ & & $18(29.0)$ & $25(55.6)$ & \\
\hline N1 & $11(27.5)$ & $6(46.2)$ & & $14(37.8)$ & $13(33.3)$ & & $17(27.4)$ & $9(20.0)$ & \\
\hline N2 & $19(47.5)$ & $2(15.4)$ & & $11(29.7)$ & $9(23.1)$ & & $27(43.5)$ & $11(24.4)$ & \\
\hline Pathological TNM stage & & & 0.349 & & & 0.472 & & & 0.018 \\
\hline I & 0 & 0 & & $1(2.7)$ & $3(7.7)$ & & $5(8.1)$ & $5(11.1)$ & \\
\hline II & $10(25.0)$ & $5(38.5)$ & & $11(29.7)$ & $14(35.9)$ & & $13(21.0)$ & $20(44.4)$ & \\
\hline III & $30(75.0)$ & $8(61.5)$ & & 25 (67.6) & $22(56.4)$ & & $44(71.0)$ & $20(44.4)$ & \\
\hline MSI status & & & 0.982 & & & 0.264 & & & 0.879 \\
\hline MSS & 37 (92.5) & $12(92.3)$ & & 35 (94.6) & 34 (87.2) & & 57 (91.9) & 41 (91.1) & \\
\hline MSI-high & $3(7.5)$ & $1(7.7)$ & & $2(5.4)$ & $5(12.8)$ & & $5(8.1)$ & $4(8.9)$ & \\
\hline
\end{tabular}

MSI: microsatellite instability; MSS: microsatellite stable; T: tumor; N: node; bold: statistically significant.

Regarding right-sided colon cancer, there was no significant difference in clinicopathological features and genetic mutations between patients with early and late recurrence, as indicated in Table 2. For left-sided colon cancer, however, more patients with early recurrence had PIK3CA and NRAS mutations than patients with late recurrence. For rectal cancer, patients with early recurrence tended to have advanced pathological $\mathrm{N}$ categories and TNM stage and to carry APC mutations compared with patients with late recurrence.

As illustrated in Figure 1 and Table 1, the gene most commonly mutated in patients with early or late recurrence was KRAS, followed by TP53, APC, and PIK3CA. As above, the most commonly mutated gene in those with right-sided colon cancer and early recurrence was KRAS, followed by TP53, APC, and PIK3CA (Figure 2A and Table 2), though the most commonly mutated gene in those with late recurrence was KRAS, followed by TP53, and PIK3CA. In left-sided colon cancer, the most commonly mutated gene in those with early recurrence was KRAS, followed by TP53, PI3KCA, and APC, and that in those with late recurrence was KRAS, followed by TP53, APC, and PIK3CA (Figure 2B and Table 2). In rectal cancer, the most commonly mutated genes in patients with early recurrence were TP53 and APC, followed by KRAS and PIK3CA (Figure 2C and Table 3). In brief, the four most commonly mutated genes were the same in those with early and late recurrence.

As indicated in Table 3, multiple-site recurrence among those with early recurrence was associated with a higher rate of NRAS mutations than single-site recurrence. Conversely, there was no difference in genetic mutations between those with single-site recurrence and those with multiple-site recurrence among patients with late recurrence. 


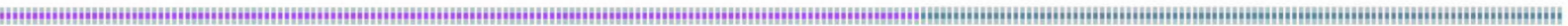

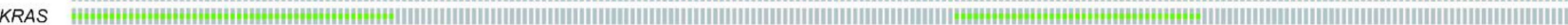
СRA

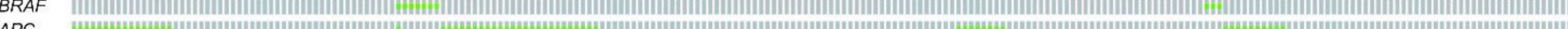
APC SMAD4 TGF TP53 PIK3CA

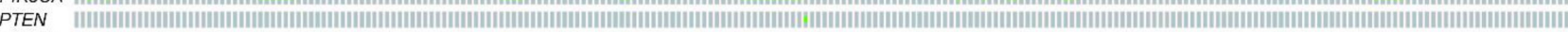
FBXW7

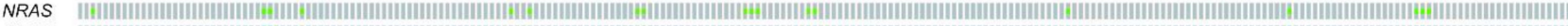

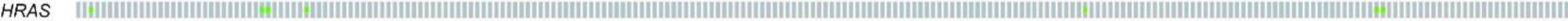

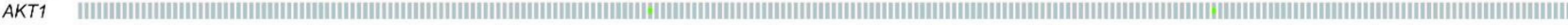

Early recurrence $\quad$ Late recurrence $\quad$ Mutation $\quad$ No alteration

Figure 1. The oncoprint of genetic mutations for all assessed colorectal cancer (CRC) patients with early and late recurrence.

(A)

\begin{tabular}{|c|c|}
\hline & Early recurrence \\
\hline & 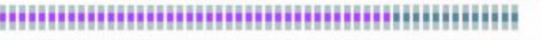 \\
\hline KRAS & 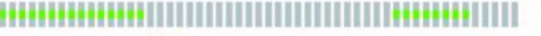 \\
\hline BRAF & 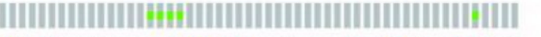 \\
\hline$A P C$ & 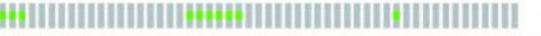 \\
\hline SMAD4 & 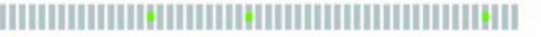 \\
\hline$T G F \beta$ & 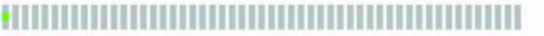 \\
\hline TP53 & 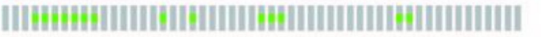 \\
\hline $3 C A$ & 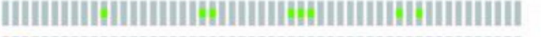 \\
\hline FBXW7 & 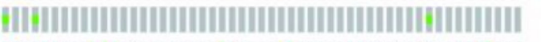 \\
\hline VRAS & 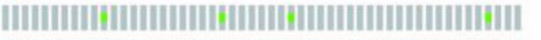 \\
\hline & ||||||||||||||||||||||||||||| \\
\hline & |||||||||||||||||||||||||||||||||||||||||||||||||||||||| \\
\hline
\end{tabular}

(B)

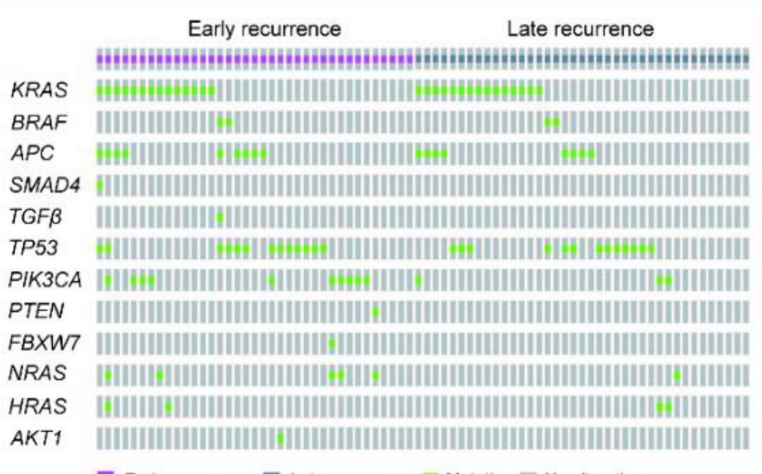

Early recurrence [n Late recurrence mutation

(C)

\begin{tabular}{|c|c|}
\hline & Early recurrence \\
\hline & 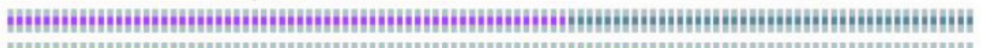 \\
\hline KRAS & 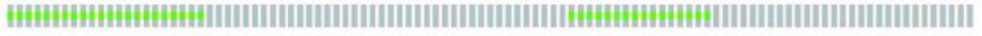 \\
\hline BRAF & 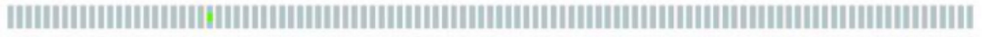 \\
\hline$A P C$ & 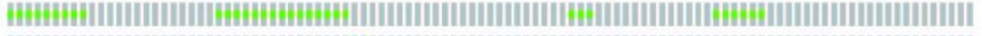 \\
\hline SMAD4 & 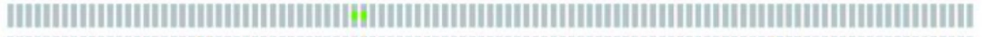 \\
\hline TGF $\beta$ & 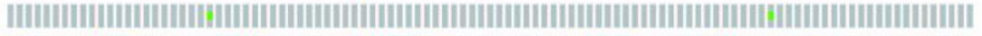 \\
\hline TP53 & 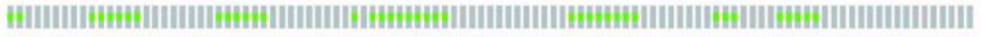 \\
\hline PIK $3 C A$ & 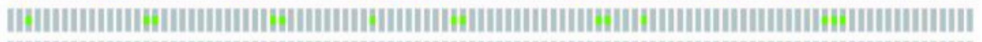 \\
\hline FBXW7 & 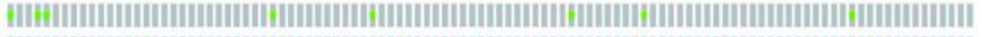 \\
\hline NRAS & 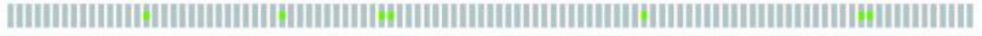 \\
\hline HRAS & 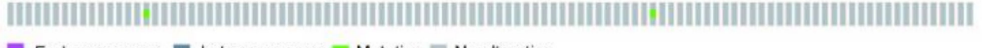 \\
\hline
\end{tabular}

Figure 2. The oncoprint of genetic alterations for patients with early and late recurrence of right-sided and left-sided CRC. The mutation profiles are shown as follows: (A) right-sided colon cancer, $(\mathbf{B})$ left-sided colon cancer, and $(\mathbf{C})$ rectal cancer. 
Table 3. The mutation spectrum of early and late recurrence in colorectal cancer stratified by number of recurrence site.

\begin{tabular}{|c|c|c|c|c|c|c|}
\hline \multirow[b]{2}{*}{ Gene } & \multicolumn{3}{|c|}{ Early Recurrence } & \multicolumn{3}{|c|}{ Late Recurrence } \\
\hline & $\begin{array}{c}\text { Single Site } \\
\text { Recurrence } \\
n=110 \\
n(\%)\end{array}$ & $\begin{array}{c}\text { Multiple Sites } \\
\text { Recurrence } \\
n=29 \\
n(\%)\end{array}$ & $p$ Value & $\begin{array}{c}\text { Single Site } \\
\text { Recurrence } \\
n=73 \\
n(\%)\end{array}$ & $\begin{array}{c}\text { Multiple Sites } \\
\text { Recurrence } \\
n=24 \\
n(\%)\end{array}$ & $p$ Value \\
\hline TP53 & $36(32.7)$ & $13(44.8)$ & 0.225 & $26(35.6)$ & $5(20.8)$ & 0.178 \\
\hline$A P C$ & $34(30.9)$ & $8(27.6)$ & 0.729 & $12(16.4)$ & $6(25.0)$ & 0.349 \\
\hline PIK3CA & $16(14.5)$ & $8(27.6)$ & 0.098 & $9(12.3)$ & $2(8.3)$ & 0.592 \\
\hline$B R A F$ & $5(4.5)$ & $2(6.9)$ & 0.607 & $3(4.1)$ & 0 & 0.313 \\
\hline KRAS & $42(38.2)$ & $9(31.0)$ & 0.477 & $32(43.8)$ & $7(29.2)$ & 0.204 \\
\hline NRAS & $7(6.4)$ & $6(20.7)$ & 0.029 & $3(4.1)$ & $2(8.3)$ & 0.417 \\
\hline HRAS & $2(1.8)$ & $2(6.9)$ & 0.146 & $3(4.1)$ & 0 & 0.313 \\
\hline FBXW7 & $6(5.5)$ & $2(6.9)$ & 0.767 & $3(4.1)$ & $1(4.2)$ & 0.990 \\
\hline PTEN & $1(0.9)$ & 0 & 0.606 & 0 & 0 & - \\
\hline SMAD4 & $4(3.6)$ & $1(3.4)$ & 0.961 & 0 & $1(4.2)$ & 0.080 \\
\hline TGF $\beta$ & $1(0.9)$ & $2(6.9)$ & 0.110 & $1(1.4)$ & 0 & 0.564 \\
\hline$A K T 1$ & $1(0.9)$ & 0 & 0.606 & $1(1.4)$ & 0 & 0.564 \\
\hline
\end{tabular}

Bold: statistically significant.

\subsection{Recurrence Patterns}

As shown in Table 4, patients with early recurrence had more liver metastases ( $44.6 \%$ vs. $33.0 \%, p=0.048$ ) and fewer metastases in other organs (other than local and the liver, lung, peritoneum, and bone) than those with late recurrence ( $4.3 \%$ vs. $15.5 \%, p=0.003)$. For right-sided and left-sided colon cancer, there was no significant difference in the recurrence pattern between patients with early and late recurrence. With regard to rectal cancer, patients with late recurrence were more likely to have recurrence in other organs (other than local and the liver, lung, peritoneum, and bone) and multiple-site recurrence ( $33.3 \%$ vs. $12.9 \%, p=0.011$ ) than patients with early recurrence.

\subsection{Survival Analysis}

As depicted in Figure 3A, patients with early recurrence had a worse five-year OS rate than those with late recurrence ( $38.2 \%$ vs. $65.9 \%, p<0.001)$. For right-sided colon cancer, those with early recurrence had a worse five-year OS rate than those with late recurrence $(29.7 \%$ vs. $80.8 \%, p=0.004$, Figure $3 \mathrm{~B})$, although there was no significant difference in the five-year OS rate between patients with early and late recurrence for left-sided colon cancer $(47.0 \%$ vs. $64.9 \%, p=0.134$, Figure 3 C). Regarding rectal cancer, patients with early recurrence had a significantly worse five-year OS rate than those with late recurrence $(39.1 \%$ vs. $62.0 \%, p=0.030$, Figure 3D).

As shown in Table 5, univariate analysis results revealed five covariates to be significantly correlated with OS: age, pathological $\mathrm{N}$ category, early recurrence, and $B R A F$ and NRAS mutations. The five covariates were included in multivariate analysis, with age, early recurrence, and $B R A F$ and $N R A S$ mutations remaining independent prognostic factors affecting OS. 
Table 4. Recurrence pattern of colorectal cancer stratified by tumor location.

\begin{tabular}{|c|c|c|c|c|c|c|c|c|c|c|c|c|}
\hline \multirow{2}{*}{$\begin{array}{l}\text { Metastatic } \\
\text { Pattern }\end{array}$} & \multicolumn{3}{|c|}{ All CRC } & \multicolumn{3}{|c|}{ Right-Sided Colon Cancer } & \multicolumn{3}{|c|}{ Left-Sided Colon Cancer } & \multicolumn{3}{|c|}{ Rectal Cancer } \\
\hline & $\begin{array}{c}\text { Early } \\
\text { Recurrence } \\
n=139 \\
n(\%)\end{array}$ & $\begin{array}{c}\text { Late } \\
\text { Recurrence } \\
n=97 \\
n(\%)\end{array}$ & $p$ Value & $\begin{array}{c}\text { Early } \\
\text { Recurrence } \\
n=40 \\
n(\%)\end{array}$ & $\begin{array}{c}\text { Late } \\
\text { Recurrence } \\
n=13 \\
n(\%)\end{array}$ & $p$ Value & $\begin{array}{c}\text { Early } \\
\text { Recurrence } \\
n=37 \\
n(\%)\end{array}$ & $\begin{array}{c}\text { Late } \\
\text { Recurrence } \\
n=39 \\
n(\%)\end{array}$ & $p$ Value & $\begin{array}{c}\text { Early } \\
\text { Recurrence } \\
n=62 \\
n(\%)\end{array}$ & $\begin{array}{c}\text { Late } \\
\text { Recurrence } \\
n=45 \\
n(\%)\end{array}$ & $p$ Value \\
\hline Local & $18(12.9)$ & $16(16.5)$ & 0.445 & $5(12.5)$ & 0 & 0.180 & $2(5.4)$ & $2(5.1)$ & 0.957 & $11(17.7)$ & $14(31.1)$ & 0.107 \\
\hline Liver & $62(44.6)$ & $32(33.0)$ & 0.048 & $16(40.0)$ & $3(23.1)$ & 0.269 & $18(48.6)$ & $15(38.5)$ & 0.370 & $28(45.2)$ & $14(31.1)$ & 0.142 \\
\hline Lung & $50(36.0)$ & $45(46.4)$ & 0.108 & $12(30.0)$ & $6(46.2)$ & 0.285 & $15(40.5)$ & $16(41.0)$ & 0.966 & $23(37.1)$ & $23(51.1)$ & 0.148 \\
\hline Peritoneum & $30(21.6)$ & $14(14.4)$ & 0.165 & $16(40.0)$ & $2(15.4)$ & 0.104 & 7 (18.9) & $9(23.1)$ & 0.657 & $7(11.3)$ & $3(6.7)$ & 0.417 \\
\hline Bone & $7(5.0)$ & $7(7.2)$ & 0.485 & $2(5.0)$ & $2(15.4)$ & 0.218 & $2(5.4)$ & $2(5.1)$ & 0.957 & $3(4.8)$ & $3(6.7)$ & 0.685 \\
\hline Others & $6(4.3)$ & $15(15.5)$ & 0.003 & $3(7.5)$ & $2(15.4)$ & 0.398 & $2(5.4)$ & $5(12.8)$ & 0.264 & $1(1.6)$ & $8(17.8)$ & 0.003 \\
\hline Recurrence site & & & 0.482 & & & 0.104 & & & 0.690 & & & 0.011 \\
\hline Single site & $110(79.1)$ & $73(75.3)$ & & $28(70.0)$ & $12(92.3)$ & & $28(75.7)$ & $31(79.5)$ & & $54(87.1)$ & $30(66.7)$ & \\
\hline Multiple sites & $29(20.9)$ & $24(24.7)$ & & $12(30.0)$ & $1(7.7)$ & & $9(24.3)$ & $8(20.5)$ & & $8(12.9)$ & $15(33.3)$ & \\
\hline
\end{tabular}

Bold: statistically significant; some patients had more than one metastatic pattern. 
(A)

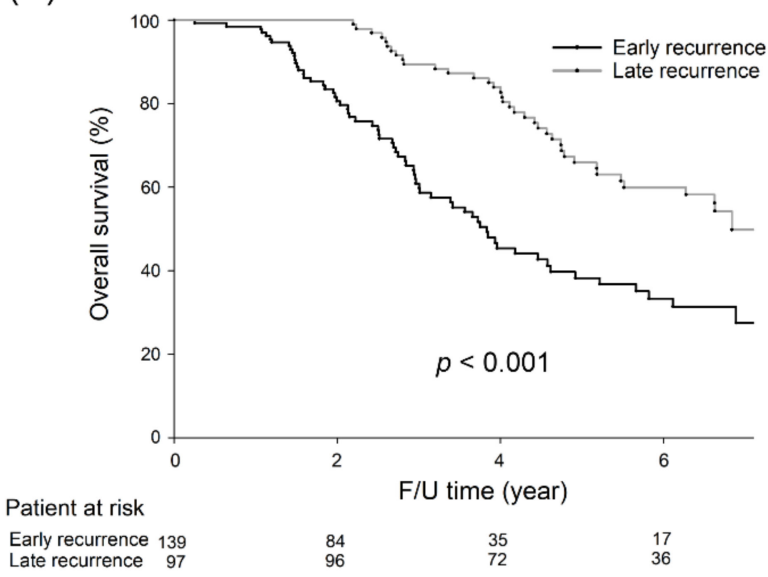

(C)

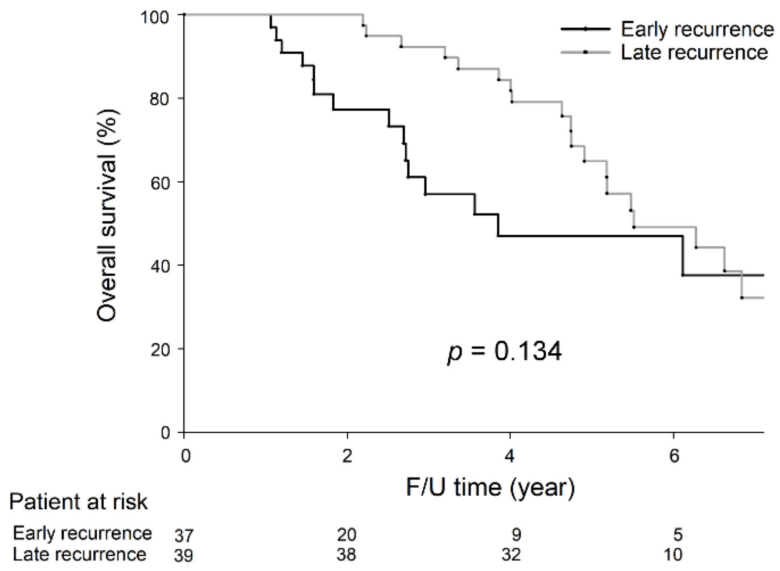

(B)

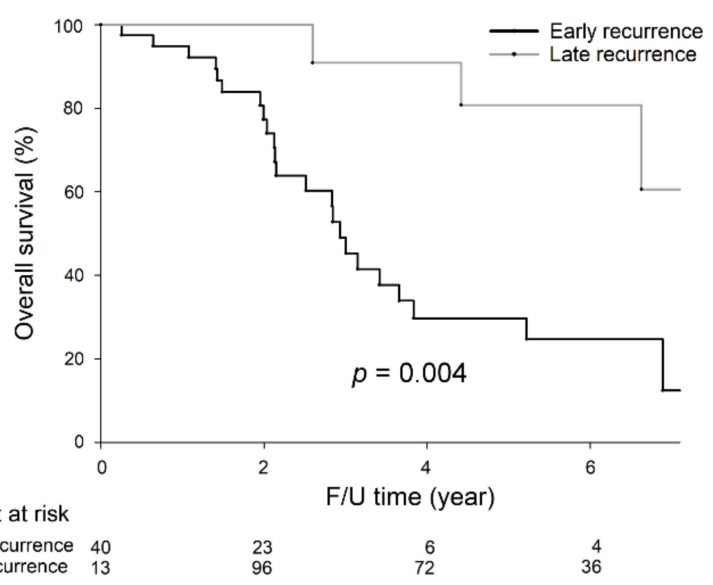

(D)

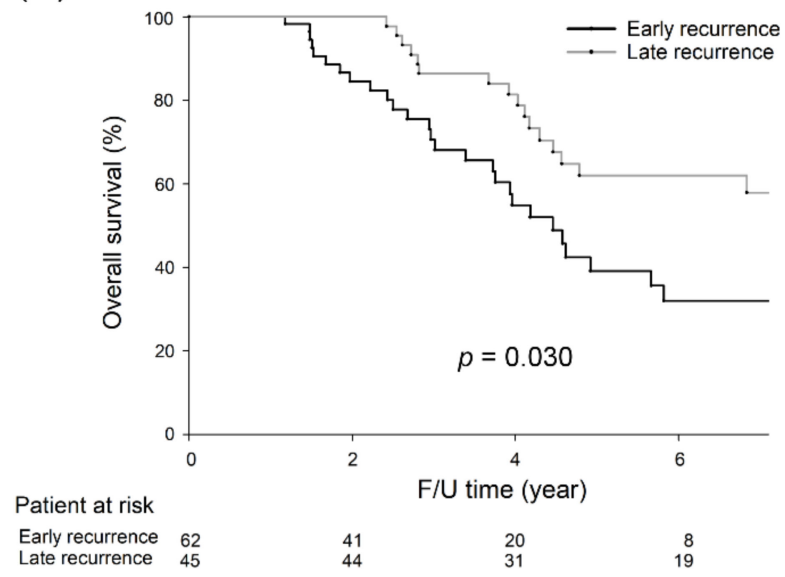

Figure 3. Five-year OS rates were significantly lower in CRC patients with early recurrence than in those with late recurrence $(38.2 \%$ vs. $65.9 \%, p<0.001)$. For right-sided colon cancer, five-year OS rates were significantly lower in CRC patients with early recurrence than in those with late recurrence $(29.7 \%$ vs. $80.8 \%, p=0.004)$. For left-sided colon cancer, five-year OS rates were not significantly different between CRC patients with early recurrence and those with late recurrence $(47.0 \%$ vs. $64.9 \%, p=0.134)$. For rectal cancer, five-year OS rates were significantly lower in CRC patients with early recurrence than in those with late recurrence $(39.1 \%$ vs. $62.0 \%, p=0.030)$. The survival curves are shown as follows: (A) all CRC patients, (B) right-sided colon cancer patients, (C) left-sided colon cancer patients, and (D) rectal cancer patients.

The univariate analysis results shown in Table 6 demonstrate that three covariates correlated significantly with survival: age and $B R A F$ and NRAS mutations. These three covariates were included in multivariate analysis, which revealed age and NRAS mutations to be associated with survival. The Cramer value is shown in Table 6. The variance inflation factor was 1.0, and the Bayesian information criterion was -961.281. 
Table 5. Univariate and multivariate cox regression analysis of overall survival in colorectal cancer with tumor recurrence.

\begin{tabular}{|c|c|c|c|c|c|c|}
\hline \multirow{2}{*}{ Variables } & \multicolumn{3}{|c|}{ Univariate Analysis } & \multicolumn{3}{|c|}{ Multivariate Analysis } \\
\hline & $\begin{array}{c}\text { Hazard } \\
\text { Ratio }\end{array}$ & $\begin{array}{l}\text { Confidence } \\
\text { Interval }\end{array}$ & $p$ Value & $\begin{array}{c}\text { Hazard } \\
\text { Ratio }\end{array}$ & $\begin{array}{c}\text { Confidence } \\
\text { Interval }\end{array}$ & $p$ Value \\
\hline Age (year) & & & 0.007 & & & $<0.001$ \\
\hline$<70$ & 1.00 & & & 1.00 & & \\
\hline$\geq 70$ & 1.66 & $1.150-2.410$ & & 2.09 & $1.409-3.088$ & \\
\hline$\overline{\text { Sex }}$ & & & 0.937 & & & \\
\hline Male & 1.00 & & & & & \\
\hline Female & 0.94 & $0.628-1.399$ & & & & \\
\hline Tumor location & & & 0.084 & & & \\
\hline Right-sided colon & 1.00 & & & & & \\
\hline Left-sided colon & 0.64 & $0.390-1.047$ & & & & \\
\hline Rectum & 0.61 & $0.386-0.962$ & & & & \\
\hline Lymphovascular invasion & 1.06 & $0.676-1.662$ & 0.800 & & & \\
\hline Pathological T category & & & 0.419 & & & \\
\hline $\mathrm{T} 1$ & 1.00 & & & & & \\
\hline $\mathrm{T} 2$ & 0.68 & $0.087-5.362$ & & & & \\
\hline $\mathrm{T} 3$ & 0.67 & $0.093-4.856$ & & & & \\
\hline $\mathrm{T} 4$ & 1.07 & $0.140-8.110$ & & & & \\
\hline Pathological N category & & & 0.031 & & & 0.163 \\
\hline N0 & 1.00 & & & 1.00 & & \\
\hline N1 & 1.56 & $1.005-2.406$ & & 1.22 & $0.777-1.900$ & \\
\hline N2 & 1.80 & $1.133-2.868$ & & 1.61 & $0.986-2.624$ & \\
\hline MSI status & & & 0.309 & & & \\
\hline MSS & 1.00 & & & & & \\
\hline MSI-high & 1.38 & $0.741-2.576$ & & & & \\
\hline Recurrence & & & $<0.001$ & & & $<0.001$ \\
\hline Early recurrence & 1.00 & & & 1.00 & & \\
\hline Late recurrence & 0.45 & $0.309-0.654$ & & 0.41 & $0.278-0.612$ & \\
\hline Recurrence site & & & 0.001 & & & 0.012 \\
\hline Single site & 1.00 & & & 1.00 & & \\
\hline Multiple sites & 2.05 & $1.358-3.104$ & & 1.84 & $1.140-2.969$ & \\
\hline Adjuvant chemotherapy & 1.26 & $0.866-1.832$ & 0.228 & & & \\
\hline Genetic mutation & & & & & & \\
\hline$B R A F$ & 2.20 & $1.065-4.524$ & 0.003 & 2.94 & $1.398-6.186$ & 0.004 \\
\hline$N R A S$ & 1.76 & $1.054-2.934$ & 0.031 & 1.59 & $0.827-3.044$ & 0.005 \\
\hline$H R A S$ & 1.31 & $0.481-3.550$ & 0.600 & & & \\
\hline TP53 & 0.88 & $0.596-1.298$ & 0.518 & & & \\
\hline$A P C$ & 0.94 & $0.613-1.437$ & 0.771 & & & \\
\hline PIK3CA & 1.50 & $0.902-2.495$ & 0.118 & & & \\
\hline KRAS & 1.17 & $0.801-1.694$ & 0.423 & & & \\
\hline FBXW7 & 1.66 & $0.840-3.293$ & 0.144 & & & \\
\hline PTEN & 4.24 & $0.583-30.787$ & 0.153 & & & \\
\hline SMAD4 & 1.39 & $0.340-5.646$ & 0.650 & & & \\
\hline$T G F \beta$ & 1.39 & $0.432-4.465$ & 0.581 & & & \\
\hline AKT1 & 1.86 & $0.459-7.570$ & 0.384 & & & \\
\hline
\end{tabular}

T: tumor; N: node; MSI: microsatellite instability; MSS: microsatellite stable; bold: statistically significant. 
Table 6. Survival analysis of clinicopathological features and mutation spectrum after curative surgery for colorectal cancer.

\begin{tabular}{|c|c|c|c|c|c|c|c|}
\hline \multirow[b]{2}{*}{ Variables } & \multicolumn{4}{|c|}{ Univariate Analysis } & \multicolumn{3}{|c|}{$\begin{array}{l}\text { Multiple Testing Correction } \\
\text { Logistic Regression }\end{array}$} \\
\hline & $\begin{array}{c}\text { Patient Alive } \\
n=120 \\
n(\%)\end{array}$ & $\begin{array}{c}\text { Patient Died } \\
n=116 \\
n(\%)\end{array}$ & $\begin{array}{l}\text { Cramer } \\
\text { Value }\end{array}$ & $p$ Value & $\begin{array}{l}\text { Odds } \\
\text { Ratio }\end{array}$ & $\begin{array}{l}\text { Confidence } \\
\text { Interval }\end{array}$ & $p$ Value \\
\hline Age (years) & & & 0.153 & 0.019 & & & 0.016 \\
\hline$<70$ & $69(57.5)$ & $49(42.2)$ & & & 1.000 & & \\
\hline$\geq 70$ & $51(42.5)$ & $67(57.8)$ & & & 1.932 & $1.132-3.297$ & \\
\hline Sex & & & 0.069 & 0.291 & & & \\
\hline Male & $76(63.3)$ & $81(69.8)$ & & & & & \\
\hline Female & $44(36.7)$ & $35(30.2)$ & & & & & \\
\hline Tumor location & & & 0.064 & 0.618 & & & \\
\hline Right-sided colon & $24(20.0)$ & $29(25.0)$ & & & & & \\
\hline Left-sided colon & $41(34.2)$ & $35(30.2)$ & & & & & \\
\hline Rectum & $55(45.8)$ & $52(44.8)$ & & & & & \\
\hline Tumor differentiation & & & 0.052 & 0.428 & & & \\
\hline Well to moderate & $112(93.3)$ & $111(95.7)$ & & & & & \\
\hline Poor & $8(6.7)$ & $5(4.3)$ & & & & & \\
\hline $\begin{array}{l}\text { Lymphovascular } \\
\text { invasion }\end{array}$ & $32(26.7)$ & $24(20.7)$ & 0.070 & 0.281 & & & \\
\hline Recurrence & & & 0.006 & 0.932 & & & \\
\hline Early recurrence & $71(59.2)$ & $68(58.6)$ & & & & & \\
\hline Late recurrence & $49(40.8)$ & $48(41.4)$ & & & & & \\
\hline Adjuvant chemotherapy & $72(60.0)$ & $70(60.3)$ & 0.004 & 0.957 & & & \\
\hline Pathological T category & & & 0.009 & 0.999 & & & \\
\hline $\mathrm{T} 1$ & $1(0.8)$ & $1(0.9)$ & & & & & \\
\hline $\mathrm{T} 2$ & $10(8.3)$ & $10(8.6)$ & & & & & \\
\hline $\mathrm{T} 3$ & $94(78.3)$ & $90(77.6)$ & & & & & \\
\hline $\mathrm{T} 4$ & $15(12.5)$ & $15(12.9)$ & & & & & \\
\hline Pathological N category & & & 0.143 & 0.089 & & & \\
\hline No & $47(39.2)$ & $40(34.5)$ & & & & & \\
\hline N1 & $28(23.3)$ & $42(36.2)$ & & & & & \\
\hline N2 & $45(37.5)$ & $34(29.3)$ & & & & & \\
\hline Pathological TNM stage & & & 0.053 & 0.717 & & & \\
\hline I & $7(5.8)$ & $7(6.0)$ & & & & & \\
\hline II & $40(33.3)$ & $33(28.4)$ & & & & & \\
\hline III & $73(60.8)$ & $76(65.5)$ & & & & & \\
\hline MSI status & & & 0.036 & 0.585 & & & \\
\hline MSS & $111(92.5)$ & $105(90.5)$ & & & & & \\
\hline MSI-high & $9(7.5)$ & $11(9.5)$ & & & & & \\
\hline \multicolumn{8}{|l|}{ Genetic mutations } \\
\hline TP53 & $42(35.0)$ & $38(32.8)$ & 0.024 & 0.716 & & & \\
\hline$A P C$ & $32(26.7)$ & $28(24.1)$ & 0.029 & 0.656 & & & \\
\hline PIK $3 C A$ & $17(14.2)$ & $18(15.5)$ & 0.019 & 0.770 & & & \\
\hline$B R A F$ & $2(1.7)$ & $8(6.9)$ & 0.130 & 0.046 & 4.806 & $0.983-23.507$ & 0.053 \\
\hline$K R A S$ & $43(35.8)$ & $47(40.5)$ & 0.048 & 0.459 & & & \\
\hline NRAS & $3(2.5)$ & $15(12.9)$ & 0.196 & 0.003 & 6.682 & $1.854-24.084$ & 0.004 \\
\hline HRAS & $3(2.5)$ & $4(3.4)$ & 0.028 & 0.668 & & & \\
\hline FBXW7 & $3(2.5)$ & $9(7.8)$ & 0.120 & 0.066 & & & \\
\hline PTEN & 0 & $1(0.9)$ & 0.066 & 0.308 & & & \\
\hline SMAD4 & $4(3.3)$ & $2(1.7)$ & 0.051 & 0.432 & & & \\
\hline TGF $\beta$ & $1(0.8)$ & $3(2.6)$ & 0.068 & 0.297 & & & \\
\hline AKT1 & 0 & $2(1.7)$ & 0.094 & 0.149 & & & \\
\hline
\end{tabular}

MSI: microsatellite instability; MSS: microsatellite stable; TNM: tumor, node, metastasis; bold: statistically significant. 
Overall, five-year postrecurrence survival rates were not significantly different between CRC patients with early recurrence and those with late recurrence (Figure $4 \mathrm{~A} ; 32.4 \%$ vs. $32.8 \%, p=0.802$ ). Furthermore, the five-year postrecurrence survival rates did not differ significantly among patients with early recurrence and those with late recurrence of right-sided colon cancer $(24.9 \%$ vs. $33.7 \%, p=0.366$, Figure $4 \mathrm{~B})$, left-sided colon cancer ( $40.6 \%$ vs. $29.9 \%, p=0.401$, Figure 4 C), and rectal cancer $(32.0 \%$ vs. $32.4 \%, p=0.594$, Figure 4D).

(A)

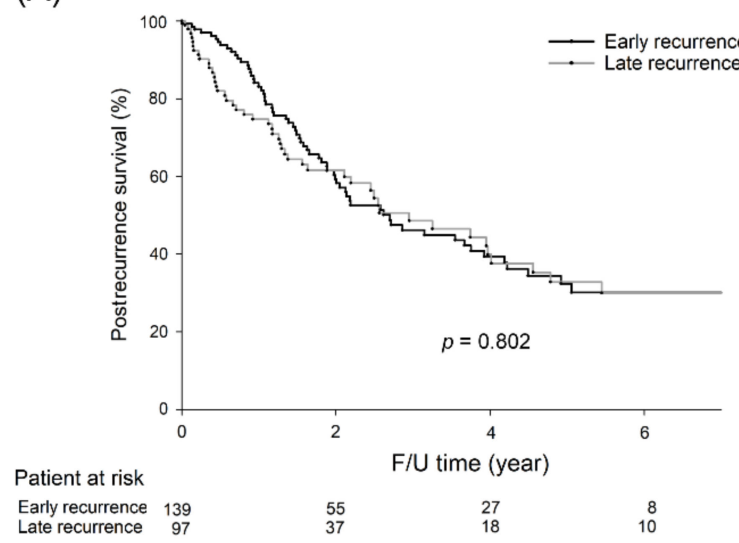

(C)

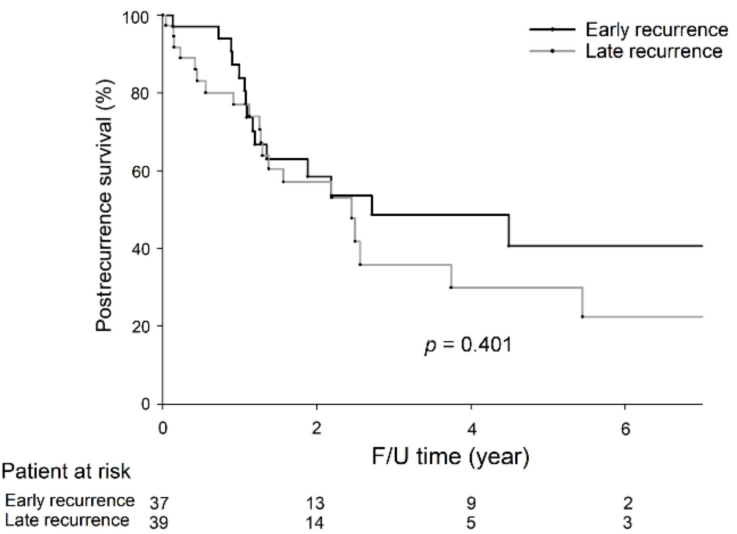

(B)

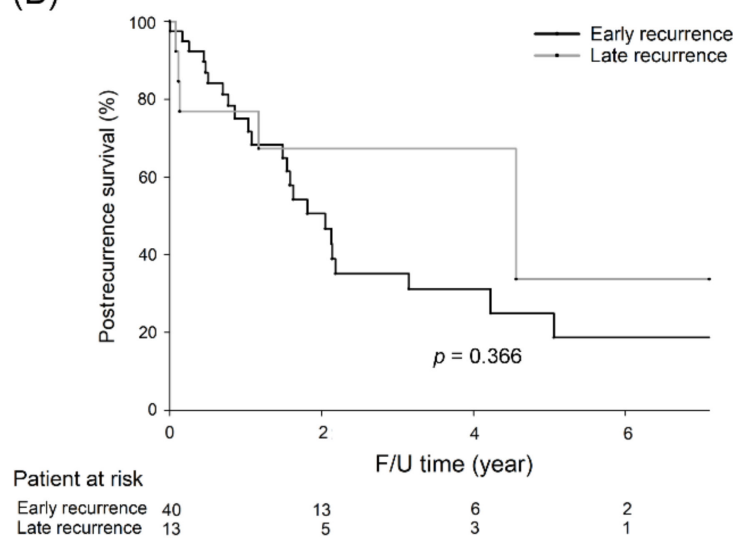

(D)

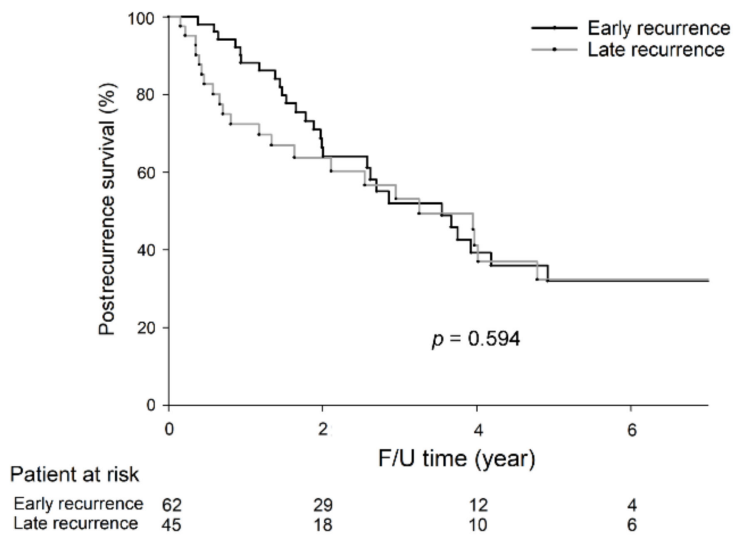

Figure 4. Five-year postrecurrence survival rates were not significantly different between CRC patients with early recurrence and those with late recurrence $(32.4 \%$ vs. $32.8 \%, p=0.802)$. For right-sided colon cancer, five-year postrecurrence rates were not significantly different between patients with early recurrence and patients with late recurrence $(24.9 \%$ vs. $33.7 \%$, $p=0.366$ ). For left-sided colon cancer, five-year postrecurrence rates were not significantly different between patients with early recurrence and patients with late recurrence ( $40.6 \%$ vs. $29.9 \%, p=0.401)$. For rectal cancer, five-year postrecurrence rates were not significantly different between patients with early recurrence and patients with late recurrence ( $32.0 \% \mathrm{vs}$. $32.4 \%, p=0.594)$. The survival curves are shown as follows: (A) all CRC patients, (B) right-sided colon cancer patients, (C) left-sided colon cancer patients, and (D) rectal cancer patients.

\section{Discussion}

To the best of our knowledge, the present study includes the largest population investigated for genetic alterations between CRC patients with early and late recurrence. The novel finding of the present study is that $A P C$ mutations were more common in CRC patients with early recurrence than in those with late recurrence, especially among those with rectal cancer. For patients with early recurrence, multiple-site recurrence was associated with a higher rate of NRAS mutations than single-site recurrence. Compared to 
late recurrence, early recurrence was an independent prognostic factor in CRC and was associated with recurrence, as well as with a poorer prognosis.

The major difference between the present study and others is that our results demonstrated that more CRC patients with early recurrence carried APC mutations than those with late recurrence, though other studies have shown that early recurrence was associated with more KRAS mutations than late recurrence. In the present study, the KRAS gene was among the top mutated genes in patients with both early and late recurrence, regardless of tumor location. It seems that KRAS mutations play an important role in CRC recurrence. Mutations in APC, KRAS, and TP53 were detected in the cell-free DNA of CRC patients, indicating that these genes may serve as a tool for the early detection of tumor recurrence [13]. In addition, the top four mutated genes in our cohort of CRC patients with tumor recurrence included KRAS, TP53, APC, and PIK3CA, and mutations in these genes were observed in those with both early and late recurrence. Based on our results, physicians should be aware of tumor recurrence in patients with mutations in the above genes; for patients with APC mutations, surveillance for tumor recurrence is necessary, especially in the first few years after surgery.

Our results showed that patients with right-sided colon cancer were more likely to develop early recurrence than were those with left-sided colon cancer or rectal cancer, which was similar to the study of Eisenberg et al. [14]. In addition, patients with early recurrence were more likely to develop liver metastasis. We further investigated the correlation between $A P C$ mutations and recurrence patterns in our cohort of patients with early recurrence, and found that those with early recurrence and APC mutations were more likely to develop lung metastasis than those without APC mutations ( $50.0 \%$ vs. $29.9 \%$, $p=0.023$ ). A higher risk to liver metastasis has been reported for CRC with APC mutations, and $A P C$ mutations are frequently detected in lung metastasis tissues $[15,16]$. It seems that $A P C$ mutations play an important role in early recurrence and hematogenous metastasis in CRC. Nevertheless, when a recurrence involves the liver within two years of surgery, it is possible that those liver metastases are microscopic metastases not detected at the time of surgery. Overall, APC mutations might be associated with silent liver metastases rather than with early recurrence.

The association of APC mutations and a hereditary cancer syndrome of familial adenomatous polyposis (FAP) is well known. APC is a tumor-suppressor gene that plays a key role in the earliest step of CRC carcinogenesis. It encodes the APC protein, which is the main component of the $\beta$-catenin destruction complex involved in suppression of the Wnt $/ \beta$-catenin pathway. Loss of APC function prevents the formation of this complex, resulting in the accumulation of $\beta$-catenin in the cytoplasm, which is later translocated to the nucleus, where it binds to TCF/LEF transcription factors. A study using CRC organoids enriched with cancer stem cells showed that noncanonical Hedgehog signaling is a positive regulator of the Wnt pathway and is required for the survival of colon cancer stem cells [17]. In the present study, among $236 \mathrm{CRC}$ patients with tumor recurrence, the frequency of APC mutations was $37.5 \%$ and $24.5 \%$ in those with and without hereditary colorectal cancer, respectively $(p=0.251)$. Among the 220 patients without hereditary colorectal cancer, those with early recurrence more often had APC mutations than those with late recurrence $(29.7 \%$ vs. $17.4 \%, p=0.037$ ). However, there was no significant difference in the frequency of $A P C$ mutations between patients with early recurrence and those with late recurrence among the 16 patients with hereditary colorectal cancer $(36.4 \%$ vs. $40.0 \%, p=0.889)$. In general, $A P C$ mutations appear to be associated with early tumor recurrence in patients without hereditary colorectal cancer. The detailed mechanism is unclear, and further in vivo and in vitro studies are required to validate our findings.

$A P C$, a tumor-suppressor gene located on chromosome 5q21-q22, is involved in CRC carcinogenesis, and Wnt/ $\beta$-catenin signaling is affected by APC mutation [18]. For example, an APC-knockout CRC mouse model revealed that restoration of $A P C$ function promoted cell differentiation and sustained tumor regression [19]. Nonetheless, the toxicity of Wnt pathway inhibitors to normal intestinal epithelium limits their clinical application [20]. 
The small molecule, truncated APC selective inhibitor (TASIN-1), can kill CRC cells with $A P C$ truncations while sparing wild-type $A P C$ cells [21]. In addition, in vivo studies have demonstrated that TASIN-1 can inhibit the growth of APC-truncated CRC cells without deleterious effects on the normal colonic epithelium, although it is not currently under clinical application.

Our results showed that $A P C$ mutations were involved in early recurrence but were not associated with OS, and that $B R A F$ and NRAS are independent risk factors for reduced survival. The mechanisms of carcinogenesis related to these genes likely differ. In general, approximately $15-30 \%$ of CRCs arise from serrated lesions, and these lesions are characterized by genetic (BRAF or KRAS mutations) and epigenetic ( $\mathrm{CpG}$ island methylator phenotype) alterations. Indeed, they are distinct from tumors arising from the conventional chromosomal instability pathway (CIN) pathway, and rarely present truncating APC mutations [22]. BRAF and KRAS mutations are considered drivers of the formation of serrated colorectal cancers [23]. Previous studies have also shown that BRAF and NRAS mutations are associated with shorter OS [24-26]. Based on our analysis, their prognostic roles are not related to the timing of recurrence.

Although our results demonstrated that among left-sided colon cancer patients, those with early recurrence were more likely to carry PIK3CA and NRAS mutations than those with late recurrence, the number of patients was small, and selection bias might have occurred. BRAF and NRAS mutations were found to be independent prognostic factors of poor OS in our cohort of CRC patients with tumor recurrence. It has been reported that $R A S$ mutation and multiple-site recurrence are independent predictors of poor survival [27]. In addition, CRC patients with BRAF or NRAS mutation are resistant to anti-EGFR therapy [28]. For our cohort of CRC patients with early recurrence, those with multiple-site recurrence had more NRAS mutations than those with single-site recurrence, which has not yet been reported. Among those with left-sided colon cancer in our cohort, approximately $16.2 \%$ of the patients in our cohort with early recurrence harbored NRAS mutations. Consequently, anti-EGFR therapy may not be helpful for these subgroups of patients.

For CRC, few biomarkers have been introduced for treatment, including RAS and $B R A F$ mutations, and MSI and CIMP status. Guinney et al. [29] classified CRC into four consensus molecular subtypes (CMSs): CMS1 tumors characterized by increased expression of genes associated with a diffuse immune infiltrate and features of MSI CRC; CMS2 tumors with strong upregulation of WNT and MYC downstream targets; CMS3 tumors with metabolic pathway dysregulation and KRAS mutations; and CMS4 tumors with clear upregulation of genes involved in the epithelial mesenchymal transition and associated with the activation of TGF $\beta$ signaling, angiogenesis, matrix-remodeling pathways, and the complement inflammatory system. However, these classifications do not predict drug response, limiting their clinical application. To identify biomarkers and predict drug sensitivity, the OncoTrack consortium developed preclinical models using a large biobank of CRC tumors, organoids, and xenografts [30]. By linking molecular profiles with drugsensitivity patterns, a novel classification outperforming $R A S / R A F$ mutations in predicting sensitivity to the EGFR inhibitor cetuximab was identified.

In the present study, multivariate analysis showed that old age, early recurrence, multiple-site recurrence, and $B R A F$ and NRAS mutations were associated with poor prognosis. All these factors potentially influence prognosis. In addition to the traditional adenoma-carcinoma sequence and serrated pathway, the inflammatory pathway plays a major role in colorectal carcinogenesis [1]. In patients with inflammatory bowel disease (IBD), an estimated 2.4-fold higher risk of CRC (95\% CI 2.1-2.7) compared with the general population has been reported for an average follow-up of 14 years [31]. The timing and frequency of molecular events in the inflammatory pathway are also distinct from the adenoma-carcinoma sequence, with TP53 mutations as an early event and infrequent APC mutations late in carcinogenesis [32]. Due to the very low incidence of IBD and IBD-related CRC in Taiwan [33], data regarding IBD-related CRC in our study cohort were 
not available, and we could not elucidate the influence of the inflammatory pathway on tumor recurrence.

There are limitations to the present study. This is a retrospective study, and only patients with tumor tissue available in the biobank were enrolled; thus, selection bias exists. Despite the significant difference observed for some genetic mutations, bias may also exist due to the small sample size and low mutation rate. To validate our results, more patients from different countries and of different races need to be studied.

\section{Conclusions}

Our results showed that CRC patients with early recurrence had worse OS, more liver metastases, and more $A P C$ mutations than those with late recurrence. Postrecurrence survival rates were not significantly different between patients with early and late recurrence.

Author Contributions: Conceptualization, Y.-T.L., S.-C.C., P.-C.L., C.-C.L., H.-H.L., S.-C.H., C.-H.L., W.-Y.L., W.-S.C., J.-K.J., J.-K.L. and S.-H.Y.; data curation, C.-H.L.; formal analysis, S.-C.C. and Y.-T.L.; funding acquisition, S.-C.C. and P.-C.L.; investigation, C.-H.L.; methodology, C.-H.L.; writingoriginal draft, S.-C.C. and Y.-T.L.; writing - review and editing, S.-C.C. and Y.-T.L. All authors have read and agreed to the published version of the manuscript.

Funding: This study was supported by the Taipei Veterans General Hospital (V105C-043, V106C-027, V107C-004); Ministry of Science and Technology, Taiwan (105-2314-B-075-010-MY2); and Taipei City Hospital (10601-62-059,10701-62-050). The funding bodies did not play a role in the design of the study; in collection, analysis and interpretation of data; or in writing the manuscript.

Institutional Review Board Statement: The study was conducted according to the guidelines of the Declaration of Helsinki and approved by the Institutional Review Board of Taipei Veterans General Hospital (Number: 2019-06-011AC).

Informed Consent Statement: Informed consent was obtained from all subjects involved in the study.

Data Availability Statement: The data presented in this study are available in the article.

Conflicts of Interest: The authors declare no conflict of interests.

$\begin{array}{ll}\text { Abbreviations } \\ \text { CIN } & \text { chromosomal instability pathway } \\ \text { CMS } & \text { consensus molecular subtype } \\ \text { COSMIC } & \text { Catalogue Of Somatic Mutations In Cancer } \\ \text { CRC } & \text { colorectal cancer } \\ \text { FAP } & \text { familial adenomatous polyposis } \\ \text { FOLFOX } & \text { folinic acid, fluorouracil, oxaliplatin } \\ \text { IBD } & \text { inflammatory bowel disease } \\ \text { MSI } & \text { microsatellite instability } \\ \text { MSS } & \text { microsatellite stable } \\ \text { LVI } & \text { lymphovascular invasion } \\ \text { OS } & \text { overall survival } \\ \text { TASIN-1 } & \text { truncated } A P C \text { selective inhibitor } \\ \text { TNM } & \text { tumor, node, metastasis }\end{array}$

\section{References}

1. Keum, N.; Giovannucci, E. Global burden of colorectal cancer: Emerging trends, risk factors and prevention strategies. Nat. Rev. Gastroenterol. Hepatol. 2019, 16, 713-732. [CrossRef] [PubMed]

2. Ministry of Health and Welfare, Executive Yuan, Taiwan, Republic of China. Taiwan Health and Welfare Report. 2019; pp. 19-20. Available online: https:/ / www.mohw.gov.tw/dl-60711-55f2159f-11a6-4c38-8438-08c8367f0d53.html (accessed on 3 March 2021).

3. Graham, R.A.; Wang, S.; Catalano, P.J.; Haller, D.G. Postsurgical surveillance of colon cancer: Preliminary cost analysis of physician examination, carcinoembryonic antigen testing, chest x-ray, and colonoscopy. Ann. Surg. 1998, 228, 59-63. [CrossRef] [PubMed] 
4. Kjeldsen, B.J.; Kronborg, O.; Fenger, C.; Jorgensen, O.D. A prospective randomized study of follow-up after radical surgery for colorectal cancer. Br. J. Surg. 1997, 84, 66-69.

5. Aghili, M.; Izadi, S.; Madani, H.; Mortazavi, H. Clinical and pathological evaluation of patients with early and late recurrence of colorectal cancer. Asia Pac. J. Clin. Oncol. 2010, 6, 35-41. [CrossRef]

6. Bozkurt, O.; Inanc, M.; Turkmen, E.; Karaca, H.; Berk, V.; Duran, A.O.; Ozaslan, E.; Ucar, M.; Hacibekiroglu, I.; Eker, B.; et al. Clinicopathological characteristics and prognosis of patients according to recurrence time after curative resection for colorectal cancer. Asian Pac. J. Cancer Prev. 2014, 15, 9277-9281. [CrossRef]

7. Lan, Y.T.; Chang, S.C.; Yang, S.H.; Lin, C.C.; Wang, H.S.; Jiang, J.K.; Chen, W.S.; Lin, T.C.; Chiou, S.H.; Lin, J.K. Comparison of clinicopathological characteristics and prognosis between early and late recurrence after curative surgery for colorectal cancer. Am. J. Surg. 2014, 207, 922-930. [CrossRef]

8. Wiesmueller, F.; Schuetz, R.; Langheinrich, M.; Brunner, M.; Weber, G.F.; Grützmann, R.; Merkel, S.; Krautz, C. Defining early recurrence in patients with resected primary colorectal carcinoma and its respective risk factors. Int. J. Colorectal Dis. 2021. [CrossRef]

9. Cancer Genome Atlas Network. Comprehensive molecular characterization of human colon and rectal cancer. Nature 2012, 487, 330-337. [CrossRef]

10. Lin, J.K.; Lin, P.C.; Lin, C.H.; Jiang, J.K.; Yang, S.H.; Liang, W.Y.; Chen, W.S.; Chang, S.C. Clinical relevance of alterations in quantity and quality of plasma DNA in colorectal cancer patients: Based on the mutation spectra detected in primary tumors. Ann. Surg. Oncol. 2014, 21, 680-686. [CrossRef]

11. Hsu, Y.L.; Lin, C.C.; Jiang, J.K.; Lin, H.H.; Lan, Y.T.; Wang, H.S.; Yang, S.H.; Chen, W.S.; Lin, T.C.; Lin, J.K.; et al. Clinicopathological and molecular differences in colorectal cancer according to location. Int. J. Biol. Markers 2019, 34, 47-53. [CrossRef]

12. Boland, C.R.; Thibodeau, S.N.; Hamilton, S.R.; Sidransky, D.; Eshleman, J.R.; Burt, R.W.; Meltzer, S.J.; Rodriguez-Bigas, M.A.; Fodde, R.; Ranzani, G.N.; et al. A National Cancer Institute Workshop on Microsatellite Instability for cancer detection and familial predisposition: Development of international criteria for the determination of microsatellite instability in colorectal cancer. Cancer Res. 1998, 58, 5248-5257.

13. Wang, J.Y.; Hsieh, J.S.; Chang, M.Y.; Huang, T.J.; Chen, F.M.; Cheng, T.L.; Alexandersen, K.; Huang, Y.S.; Tzou, W.S.; Lin, S.R. Molecular detection of APC, K- ras, and p53 mutations in the serum of colorectal cancer patients as circulating biomarkers. World J. Surg. 2004, 28, 721-726. [CrossRef]

14. Eisenberg, B.; Decosse, J.J.; Harford, F.; Michalek, J. Carcinoma of the colon and rectum: The natural history reviewed in 1704 patients. Cancer 1982, 49, 1131-1134. [CrossRef]

15. Schweiger, T.; Liebmann-Reindl, S.; Glueck, O.; Starlinger, P.; Laengle, J.; Birner, P.; Klepetko, W.; Pils, D.; Streubel, B.; Hoetzenecker, K. Mutational profile of colorectal cancer lung metastases and paired primary tumors by targeted next generation sequencing: Implications on clinical outcome after surgery. J. Thorac. Dis. 2018, 10, 6147-6157. [CrossRef]

16. Datta, J.; Smith, J.J.; Chatila, W.K.; McAuliffe, J.C.; Kandoth, C.; Vakiani, E.; Frankel, T.L.; Ganesh, K.; Wasserman, I.; Lipsyc-Sharf, M. Coaltered Ras/B-raf and TP53 Is Associated with Extremes of Survivorship and Distinct Patterns of Metastasis in Patients with Metastatic Colorectal Cancer. Clin. Cancer Res. 2020, 26, 1077-1085. [CrossRef]

17. Regan, J.L.; Schumacher, D.; Staudte, S.; Steffen, A.; Haybaeck, J.; Keilholz, U.; Schweiger, C.; Golob-Schwarzl, N.; Mumberg, D.; Henderson, D.; et al. Non-Canonical Hedgehog Signaling Is a Positive Regulator of the WNT Pathway and Is Required for the Survival of Colon Cancer Stem Cells. Cell Rep. 2017, 21, 2813-2828. [CrossRef]

18. Zhang, L.; Shay, J.W. Multiple Roles of APC and its Therapeutic Implications in Colorectal Cancer. J. Natl. Cancer Inst. 2017, 109, 332. [CrossRef]

19. Dow, L.E.; O’Rourke, K.P.; Simon, J.; Tschaharganeh, D.F.; van Es, J.H.; Clevers, H.; Lowe, S.W. Apc Restoration Promotes Cellular Differentiation and Reestablishes Crypt Homeostasis in Colorectal Cancer. Cell 2015, 161, 1539-1552. [CrossRef]

20. Kahn, M. Can we safely target the WNT pathway? Nat. Rev. Drug Discov. 2014, 13, 513-532. [CrossRef]

21. Zhang, L.; Theodoropoulos, P.C.; Eskiocak, U.; Wang, W.; Moon, Y.A.; Posner, B.; Williams, N.S.; Wright, W.E.; Kim, S.B.; Nijhawan, D.; et al. Selective targeting of mutant adenomatous polyposis coli (APC) in colorectal cancer. Sci. Transl. Med. 2016, 8, 140. [CrossRef]

22. De Palma, F.D.E.; D'Argenio, V.; Pol, J.; Kroemer, G.; Maiuri, M.C.; Salvatore, F. The Molecular Hallmarks of the Serrated Pathway in Colorectal Cancer. Cancers 2019, 11, 1017. [CrossRef] [PubMed]

23. Patai, A.V.; Molnár, B.; Tulassay, Z.; Sipos, F. Serrated pathway: Alternative route to colorectal cancer. World J. Gastroenterol. 2013, 19, 607-615. [CrossRef] [PubMed]

24. Taieb, J.; Le Malicot, K.; Shi, Q.; Penault-Llorca, F.; Bouché, O.; Tabernero, J.; Mini, E.; Goldberg, R.M.; Folprecht, G.; Luc Van Laethem, J.; et al. Prognostic Value of BRAF and KRAS Mutations in MSI and MSS Stage III Colon Cancer. J. Natl. Cancer Inst. 2016, 109, djw272. [CrossRef]

25. Guo, T.A.; Wu, Y.C.; Tan, C.; Jin, Y.T.; Sheng, W.Q.; Cai, S.J.; Liu, F.Q.; Xu, Y. Clinicopathologic features and prognostic value of KRAS, NRAS and BRAF mutations and DNA mismatch repair status: A single-center retrospective study of 1,834 Chinese patients with Stage I-IV colorectal cancer. Int. J. Cancer. 2019, 145, 1625-1634. [CrossRef] [PubMed]

26. Schirripa, M.; Cremolini, C.; Loupakis, F.; Morvillo, M.; Bergamo, F.; Zoratto, F.; Salvatore, L.; Antoniotti, C.; Marmorino, F.; Sensi, E.; et al. Role of NRAS mutations as prognostic and predictive markers in metastatic colorectal cancer. Int. J. Cancer. 2015, 136, 83-90. [CrossRef] 
27. Lillemoe, H.A.; Kawaguchi, Y.; Passot, G.; Karagkounis, G.; Simoneau, E.; You, Y.Q.N.; Mehran, R.J.; Chun, Y.S.; Tzeng, C.W.D.; Aloia, T.A.; et al. Surgical Resection for Recurrence after Two-Stage Hepatectomy for Colorectal Liver Metastases is Feasible, is Safe, and Improves Survival. J. Gastrointest. Surg. 2019, 23, 84-92. [CrossRef]

28. Therkildsen, C.; Bergmann, T.K.; Henrichsen-Schnack, T.; Ladelund, S.; Nilbert, M. The predictive value of KRAS, NRAS, BRAF, PIK3CA and PTEN for anti-EGFR treatment in metastatic colorectal cancer: A systematic review and meta-analysis. Acta Oncol. 2014, 53, 852-864. [CrossRef]

29. Guinney, J.; Dienstmann, R.; Wang, X.; de Reyniès, A.; Schlicker, A.; Soneson, C.; Marisa, L.; Roepman, P.; Nyamundanda, G.; Angelino, P.; et al. The Consensus Molecular Subtypes of Colorectal Cancer. Nat. Med. 2015, 21, 1350-1356. [CrossRef]

30. Schütte, M.; Risch, T.; Abdavi-Azar, N.; Boehnke, K.; Schumacher, D.; Keil, M.; Yildiriman, R.; Jandrasits, C.; Borodina, T.; Amstislavskiy, V.; et al. Molecular dissection of colorectal cancer in pre-clinical models identifies biomarkers predicting sensitivity to EGFR inhibitors. Nat. Commun. 2017, 8, 14262. [CrossRef]

31. Jess, T.; Rungoe, C.; Peyrin-Biroulet, L. Risk of colorectal cancer in patients with ulcerative colitis: A meta-analysis of populationbased cohort studies. Clin. Gastroenterol Hepatol. 2012, 10, 639-645. [CrossRef]

32. Itzkowitz, S.H.; Yio, X. Inflammation and cancer IV. Colorectal cancer in inflammatory bowel disease: The role of inflammation. Am. J. Physiol. Gastrointest. Liver Physiol. 2004, 287, G7-G17. [CrossRef]

33. Wei, S.C.; Chang, T.A.; Chao, T.H.; Chen, J.S.; Chou, J.W.; Chou, Y.H.; Chuang, C.H.; Hsu, W.H.; Huang, T.Y.; Hsu, T.C.; et al. Management of Crohn's disease in Taiwan: Consensus guideline of the Taiwan Society of Inflammatory Bowel Disease. Intest. Res. 2017, 15, 285-310. [CrossRef] 